The association of localised hypertrophy with novus and varices, though rare, appears to form a definite clinical entity. The disease, when fully developed, presents three features, of which the nævus is congenital, the hypertrophy also congenital but more apparent as growth develops, whilst the varices appear later, and in some instances of the disease are small or entirely lacking. The interesting association of the three conditions, no less than the striking deformity that they produce, has given rise to a fairly considerable literature on the subject, and this literature is almost entirely of French origin. The first mention of the disease which I have been able to find is by $\mathrm{J}$. Adams, who reported a case in THE LANCET of August 7th, 1858. This case, which is reported without comment as a curiosity encountered in the course of casualty work, was evidently a typical specimen of the disease. The first important reference is the research of Trelat and Monod on Asymmetry, which appeared in 1869 . This was followed by papers by Leblanc (1897), Duplay (1898), and by a very complete article by Klippel and Trenaunay in 1900 . Grimaud took the disease as the subject of a Paris thesis in 1901.

A study of this literature shows that the association of nævus. local hypertrophy, and varicose veins is a definite one which has been encountered in all parts of the body. It has, however, been met with most frequently in the lower extremities, and very curiously the reported cases refer, almost without exception, to the right side.

The main features of the disease are as follows. The nævus is usually superficial but very extensive, with an irregular, sinuous contour. However large the area of the nævus may be it is always sharply limited by the middle line, and several cases are on record in which the nærus was arrested at the scrotal raphe. The localised overorowth of the part is usually most apparent in the bones, and has affected the bones of the skull, face, upper and lower extremities. The bones are increased in all dimensions, and the disparity of the two sides has amounted to difference of as much as four inches in the length of the lower limbs. The coexistence of dilated superficial veins is remarkable, since varicose veins are very rare in childhood. The varices vary much in number and extent in different cases, and are the least constant of the three chief features of the disease. They are usually superficial and small, seldom attaining the proportions seen in the varices of adults.

Whilst the hypertrophy, nævus, and varices constitute the three classical signs of the condition, there are often found other phenomena which are of interest as indicating the farreaching nature of the congenital fault. The skin of the hypertrophied parts is in some cases thick, rough, and dry, the condition amounting at times to an ichthyosis. The hair may be abundant and coarse in texture. The nails of the affected limb have been described as deformed, showing furrows along their length. Excessive secretion of sweat and of sebaceous material has been a striking feature of some cases. The subcutaneous tissue and the muscle have usually shared in the overgrowth of the affected part.

The origin of the disease is unknown. Many theories, ranging from maternal impression to vaso-motor paralysis, have been offered in explanation, but a review of them merely confirms the opinion that the origin of this disease is one of the many mysteries in the field of ante-natal pathology. Manchester.

\section{A CASE OF MENINGITIS}

IN WHICH THE ONLY ORGANISM THAT COULD BE CULTIVATED FROM THE CEREBRO-SPINAL FLUID DURING LIFE WAS A BACILLUS OF THE PROTEUS GROUP.

Br E. ATHOLE ROSS, M.D. Cantab., M.R.C.P. Lond., PATHULOGISI TU THE EAST LONDON HOSPITAL FOR CHILDREN, SHADWEIL.

THE following is a brief abstract of the notes in this case, to which are appended the post-mortem and clinical pathological reports.

The patient, a male child, aged 9 months, was admitted to the East London Hospital for Children on March 31st, suffering from stiff neck of two days' duration. The mother noticed it when she bathed the child. He could not keep his food down-vomited it at once. He emitted a peculiar squeal during most of the night. The child had not been well since he began teething three months before. The bowels were constipated. The mother noticed that he used " to work his eyes a lot at night." She thought this was due to fits. 'There was nothing of importance in his previous history. With regard to the family history, four children were alive out of seven. Two died from bronchitis anc croup and one from epilepsy. The father and mother were healthy.

On admission the child was well nourished, pale, and very drowsy; the head was retracted. There was marked rigidity of the neck. Kernig's sign suspicious on both sides. Abdominal reflex increased on the left side, less marked on the right. Knee-jerks were present. Fontanelle was not bulged. Discs were normal. Lumbar puncture, nil; three attempts, no fluid. On April 3rd rigidity was more marked. Head was very retracted and abdomen sunken and soft.

FIG. 1.

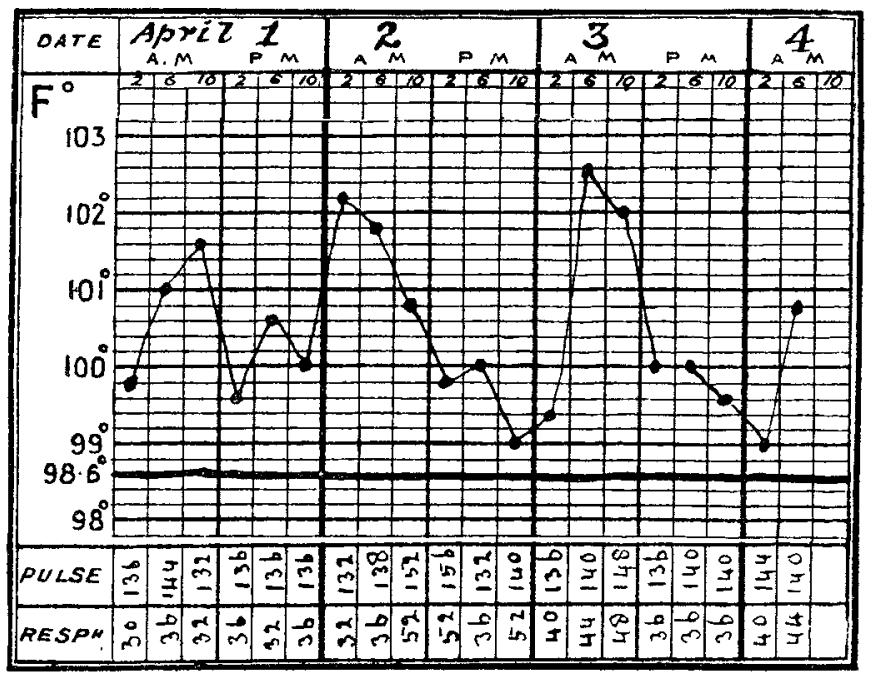

Temperature Chart.

Abdominal reflexes slight. On the 4th he was very rigid. 10 c.c. of spinal fluid were drawn off on April 3rd. Smear showed bacilli, some of which were intracellular, but most extracellular. Fontanelle bulged this morning; much worse. The patient died.

Abstract of the Post-mortem Report.

Brain: The entire surfaces at the vertex and base were covered with a thick layer of greenish-yellow pus, and the ventricles were also dilated and contained pus. The cerebral tissue was sectioned in all directions without finding any evidence of a localised abscess. Spinal cord: This thick greenish-yellow pus stripped away with the membranes and formed a layer nearly a quarter of an inch thick. Lungs: Emphysematous. Liver: Cloudy swelling and fatty change. Intestines: Both the large and small intestine showed patches of enteritis with injection and swelling of the mucous membrane. ${ }^{1}$ Middle ears : The middle ears on both sides contained pus. The right more than the left. The cerebro-spinal fluid: This was withdrawn under aseptic precautions by Dr. A. G. Hamilton in a sterile tube and carried to the laboratory, where an examination was made immediately. turbid, and contained large numbers of bacilli. Most of the organisms turbid, and contained large numbers of bacilli. Most of the organisms were extracellular, but a fair proportion were seen to be inside the cells in stained film preparations. A rlifferential count gave 93 per cent. of
polymorphonuclear cells. Bacteriology : Pure cultures of the organism were obtained on agar. The bacilli varied in length and short forms were present. Gram-negative. Agar plate: Greyish white colonies,
discrete, with a surrounding foggy haze which gradually spread over discrete, with a surrounding foggy haze which gradually spread over
the plate. Neutral red agar plate: No growth. Litmus mannite: Acid; no gas. Litmus maltose and litmus lactose: Diffuse turbidity. No acid or gas. McConkey: No change. Litmus milk: Slight acidity. No clot in seven days. Gelatin slope: Rapid liquefaction of medium (in 18 hours). Broth: Diffuse turbidity. In maltose and lactose growth was fair, but film preparations from the McConkey tube showed that the organism had died out. The growth on gelatin was examined by Gram's method, and Gram-negative bacilli were found present in pure culture. Since a slight greenish tinge was observed in the exudate post mortem the possible presence of $B$. pyocyaneus had to be considered, although neither the tint of the colonies on agar nor the odour of the broth cultures was in the least suggestive. A peptone water culture incubated for a week gave a totally negative result.

Instances of meningitis in which bacilli, other than B. tuberculosis, figure in the cerebro-spinal fluid during life are distinctly uncommon. The recognised infective agents are as follows.

1. The typho-oolon group.-The presence of a Gramnegative bacillus rapidly liquefying gelatin at once excludes this family with its many bacteriological complexities, including $\mathrm{B}$. paratyphosus $\mathrm{A}$ and $\mathrm{B}$ with the other members of the Salmonella group. The acid and gas-forming powers of $B$. proteus in the litmus sugar media are very variable,

1 These lesions may be of some importance in view of the bacteriological tindings. 
and in certain examples agree closely with a typical B. coli, while in others mere turbidity or only acid formation without gas is found. The "fogging" of agar and bile salt plate media is rather characteristic of the organism; as a rule, it grows well on both neutral red agar and on Conradi Drigalski plates, but I have notes of two instances in which a urinary B. proteus failed to grow on a neutral red and on a Conradi Drigalski plate respectively. Not much attention is given to the cultural features of $\mathrm{B}$. proteus in most of the standard bacteriological text-books. Mr. G. H. Pearson has reported a case of $\mathbf{B}$. coli meningitis. The child was an in-patient of this hospital, under the care of Dr. A. M. Gossage. Mr. Pearson also collected one or two parallel instances from the literature. Very recently Dr. E. Bellingham Smith and Dr. A. W. G. Woodforde have reported a case; they give references to the literature, and also refer to Mr. Pearson's article in THE LANCET. Both the typhoid bacillus and the $B$. enteritidis of Gaertner have been found in the cerebro-spinal fluid during life, and the diagnosis confirmed by cultural investigations.

2. Friedländer's pneumobacillus.--This organism is also a recognised cause of bacillary meningitis. The bacillus, though Gram-negative, does not liquefy gelatin, and produces in stab cultures on that medium the characteristic roundheaded nail formation. It is. moreover, an active fermenter of the litmus sugar media. A point of resemblance to B. proteus is its tendency in cultivations to form long rods as well as to exhibit short forms.

3. Influenza bacillus. - In 1892 Pfûhl first demonstrated the presence of Pfeiffer's bacillus in the meningeal exuclate; it was obtained post mortem from an adult, and also cultivated during life from the cerebro-spinal fluid of a child. Since the organism is grown with difficulty on media other than glycerine and blood agar, soon dies out, and refuses to grow on media at $22^{\circ} \mathrm{C}$, it is excluded. It moreover produces very small, translucent, fine colonies on suitable media. According to Klein it can only be kept alive on gelatin by incubating at $37^{\circ} \mathrm{C}$., thus producing an artificial liquefaction.

4. Leptothrix associated with tubercle bacillus.--As regards the latter organism, the bacillus with which we are dealing was Gram-negative and possessed no acid-fast properties. A few long bacilli were present, but nothing like a filament. and most of the organisms were short. In his leptothrical case Dr. Bellingham Smith found a turbid cerebro-spinal fluid exhibiting an intense polynuclear leucocytosis and which yielded on culture a very actively motile organism. Subsequently it was proved that the colonies consisted of filaments up to $100 \mu$ in length, and also of short actively motile bacillary forms. Both forms were Gram-negative, but repeated attempts to separate them failed. Growth was obtained on gelatin at $20^{\circ} \mathrm{C}$. withont liquefaction.

Agglutination reactions. - A tube of blood was obtained shortly before death, centrifuged, and tested. A filtered emulsion of the organism in 0.85 per cent. salt was free from clumps and showed no tendency to agglutinate; the organisms were non-motile. The usual technique of the Widal reaction was followed, with the result that the patient's own serum agglutinated the organism from his cerebro-spinal fluid well in dilutions of 1 in 10 and 1 in 20 , and less well up to 1 in 30 . The patient's serum in a dilution of 1 in 10 was entirely without action on B. typhosus, B. paratyphosus, or B. coli. My own serum, that of the laboratory attendant, and of a nurse failed to react on the organism from the cerebro-spinal fluid in dilutions higher than 1 in 8,1 in 5 , and 1 in 5 respectively. The duration of the illness at the time these tests were applied was between a week and 10 days, according to the history obtained. There was thus evidence that the patient had elaborated agglutinins in his serum during life against the organism isolated also during life from his cerebro-spinal fluid. A case of very chronic otorrhcea, under the care of Mr. W. Girling Ball, in which the B. proteus was cultivated, happened to be examined more recently, when it was found that the patient's serum possessed no agglutinative properties for his own organism. In the Croonian lectures for the present year Mr. L. S. Dudgeon refers to certain proteus exudates prepared from rabbits injected with B. proteus. On injection of these exudates into rabbits intraperitoneally the animals developed active agglutinins in their serum for $B$. proteus.
Pathogenicity. - $\mathrm{lt}$ is sometimes a little difficult to convince those who are not laboratory workers that the B. proteus possesses pathogenic properties. This is probably owing to the well-known saprophytic properties of the organism and its frequent appearance as a secondary infection in chronic otorrhoea or contaminated wounds where a drainage-tube has been in position for some time. No one who has worked at the bacteriology of the urinary tract can fail to have met with a few instances of proteus infection in which the patient's symptoms and condition left no reasonable cloubt of the powers of this organism for active mischief. The following quotation, in which I am responsible for the italics, from the Horace Dobell lecture for 1908 is very much to the point. Speaking of B. proteus, the author observes: "This group of bacteria is fonnd most commonly in the urine and intestinal tract. In the former it may be the cause of bacilluria, and may have been present for varying periods unknown to the patient. It may at any time and for some unknown reason give rise to active infection of the urinary tract, but this is not, however, of very common ocourrence. It is known that in some cases of cerebral abscess the only organism nthich is cultivated from the pus may be one of the proteus group. This fact is of great interest because in some cases of chronic middle-ear disease the discharge will be found to show the presence of this bacillus in pure culture. The proteus group serves as an excellent illustration of the all-important fact referred to elsewhere in this

FIG. 2

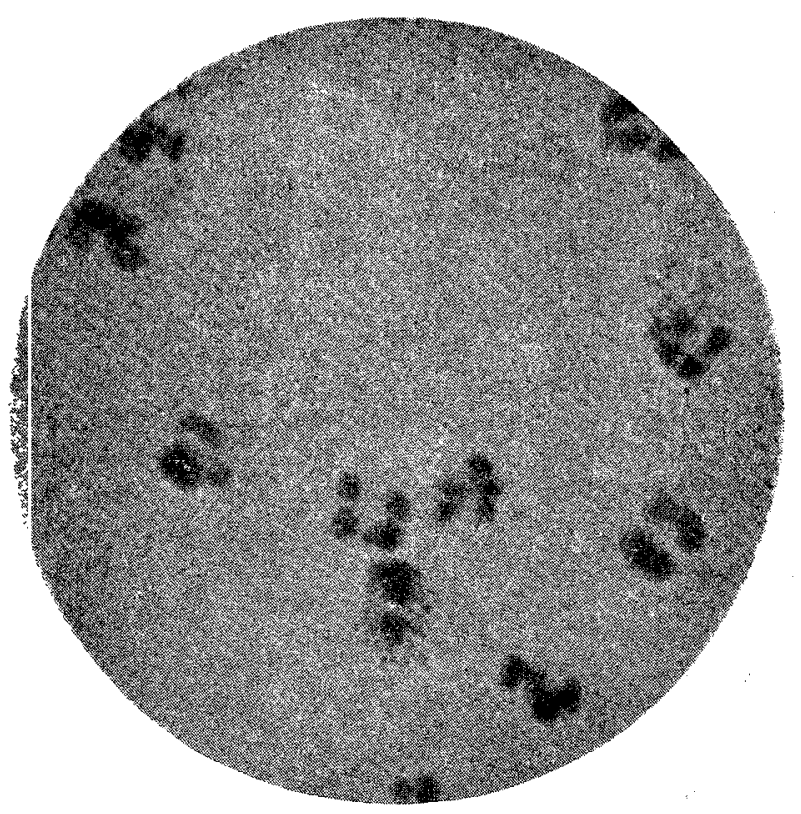

Photomicrograph of the cerebro-spinal fluid. Shows polymorphonuclear leucocytes with intracellular and free bacilli. 1/12th in. oil immersion objective. Stained carbolthionin-blue.

lecture-namely, the persistence of an organism in the tissues for indefinite periods without giving rise to any symptoms, but occasionally acting as the cause of some acute illness." As regards its pathogenic properties for the laboratory animals in the same set of Croonian lectures already referred to, Mr. Dudgeon states that: "Numerous experiments were made with cultures of the bacillus proteus inoculated from cases of cystitis and infected wounds. These cultures were all proved to be highly virulent for rabbits."

The post-mortem examination.-Owing to the unfortunate circumstance that the time of the patient's disease necessitated the performance of a necropsy on Easter Sunday, no cultural investigations were undertaken. It can only be stated that bacilli were present in large numbers in the cerebral exudate and that the pus consisted almost entirely of polymorphonuclear leucocytes (Fig. 2). There was no evidence of localised abscess, although the ventricles were full of purulent fluid. In favour of the view that an acute proteus infection of the meninges was the cause of death are : (1) the presence of the bacillus in the cerebro-spinal fluid during life, together with numerous polymorphonuclear leucocytes, some of which were phagocytic; (2) the presence of 
a certain amount of agglutinin agains this own organism in the patient's serum at the terminal stages of his illness ; (3) the acknowledged pathogenic powers of B. proteus in certain rather exceptional instances, and the proof that some strains of it are virulent for rabbits ; (4) the fact that it has been cultivated from cases of cerebral abscess; and (5) the presence of pus in both middle ears and the well-known connexion of the organism with many cases of otitis media. Against this view it may be argued that the failure to recover the organism after death and to investigate its cultural reactions affords a loophole for criticism, although that failure happened to be due to circumstances beyond my control. It can be further urged that the real infective agent was overgrown, or, so to speak, swamped by a secondary proteus infection, although personally I am of opinion that the weight of evidence is against such a supposition in this particular case. Lastly, in respect to finding pus in the middle ear, Dr. R. S. Frew informs me that he has noted pus in the middle ear in a very large percentage of all necropsies on young children at the Great Ormondstreet Hospital. If I understand him rightly, Dr. Frew does not consider that the presence of pus uncler these circumstances necassarily points to active middle-ear disease, and $I$ have myself found it present when there were no ear symptoms during life.

The film prepared from the cerebro-spinal fluid was stained by carbol-thionin, and although the cell nuclei took up the stain very well, the bacilli were much less deeply tinged ; in

FIG. 3.

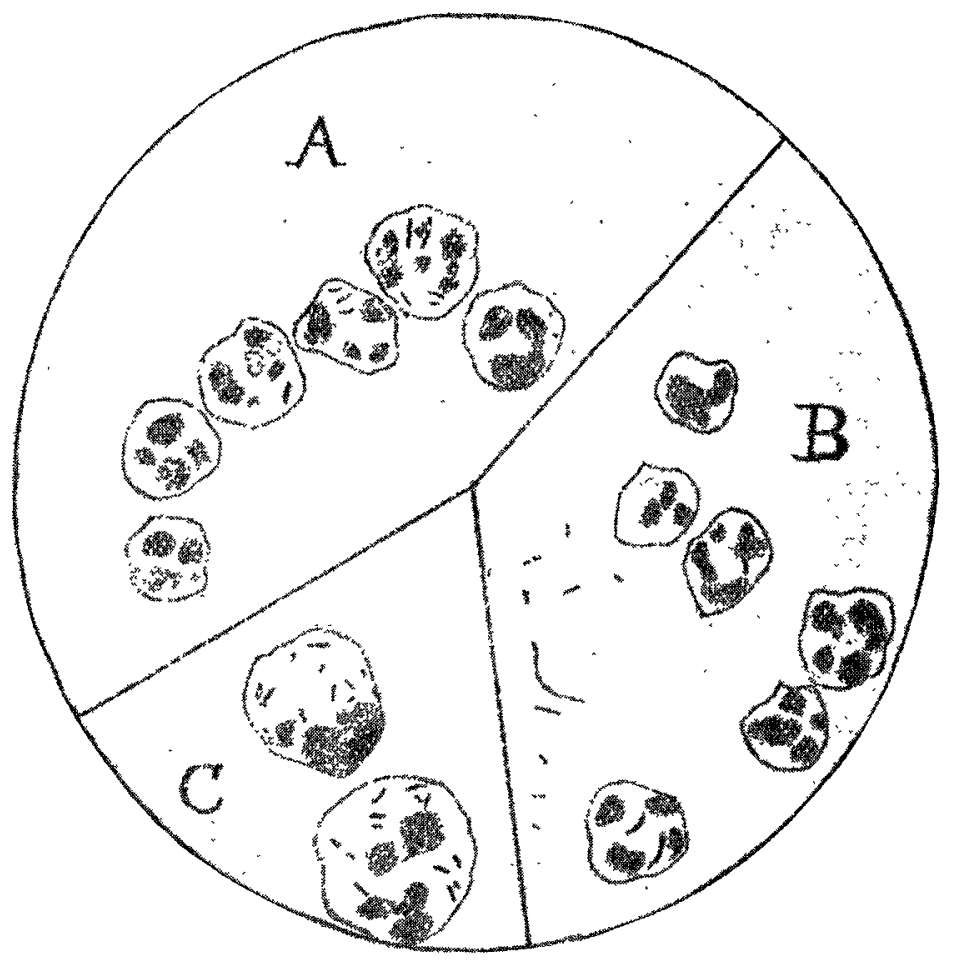

A drawing compounded of three different fields. A, Shows a chain of phagocytes. B, Bacilli lying free in the tield. c, Glutton cells. 1/12th Zeiss objective. Ocular No.6.

consequence, it was difficult to obtain a photomicrograph showing the morphological characteristics of the organisms satisfactorily. To obviate this to some extent a drawing has been prepared to show both the phagocytic action in progress and also the appearances of the bacilli (Fig. 3).

In conclusion, I have to thank both Dr. Gossage for permission to publish this case, and also Dr. A. G. Hamilton, the resident medical officer, for supplying me with the notes of the necropsy.

Bibliography.-The Latent Persistence and the Reactivation of Pathogenic Bacteria in the Body, The Horace Dobell Lecture for 1908, L. S. Dudgeon, The LAvCET, Dec. 5th, 1908, p. 1651. A Case of Pyæmia due to the Influenza Bacillus, with Multiple Arthritis and Meningitis, L. S. Dudgeon and J. E. Adams, THE LANCET, Sept. 7th, 1907, p. 684. The Croonian Lectures on the Pathology of Immunity, L. . Dudgeon, THE LANCET, June 15th (p. 1593), 22nd (p. 1671), and 29th (p. 1737), and July 6th (p. 1), 1912. A Case of Meningitis in which the B. Coli Communis was Obtained from the Cerebro-spinal Fluid, G. H. Pearson, THE LANCET, March 16th, 1912, p.722. Two Unusual Forms of Woodforde, THE LANCET, July $27 \mathrm{th}, 1912$, p. 236.

\section{A CASE OF PNEUMOCOCCAL CEREBRO- SPINAL MENINGITIS.}

By J. H. CUMMING, M.R.C.S. ENG., L.R.C.P. Lonn.

(With Remarks by Dr. HaRrington SaInsBury.)

THE patient, a male aged 12 years, was admitted to the Royal Free Hospital, under the care of Dr. Sainsbury, on Feb. 6th, 1912. There had been twitchings of the face for three months. Four days before admission severe vomiting had set in, followed by acute pain in the back of the head and neck, and in the back of the legs. The child became delirious and there was retraction of the head.

On admission the patient lay with his knees drawn up; he was delirious and the head was markedly retracted; ho complained of great pain in the back of the head and neck. Temperature $102^{\circ} \mathrm{F}$., pulse-rate 92 , respirations 28 . The vomiting continued. Physical examination revealed marked retraction of the head, no rigidity of the upper limbs, the legs somewhat rigid and flexed at the hip and knee-joints, and the abdominal wall retracted. There was no squint or evidence of affection of any of the cranial nerves. The knee-jerks were slightly increased, but there was no patellar or ankle clonus. The plantar response was indefinite, but not extensor. Kernig's sign was well marked. The abdominal reflexes were present and were equal. Nothing abnormal was detected in the heart, lungs, or abdominal organs. A lumbar puncture was made and about 3 c.c. of cerebrospinal fluid were withdrawn apparently under normal pressure. It was examined and reported on as follows :-

Colourless turbid fluid containing some blood and a trace of albumin, no reducing substance. Microscopically many leucocytes, about 80 pe cent. of which are polymorphonuclear. Direct films contain large numbers of Gram-positive diplococci, probably pneumococci. The culture has grown staphylococci, probably contamination, and some pneumococci.

On Feb. 8th the temperature fell to $97^{\circ}$, but the vomiting and headache continued, the latter being unrelieved by icebags. On this date lumbar puncture was again performed and the fluid withdrawn was examined. The report was :-

Slightly turbid fluid, a little fibrinous coagulum. Microscopically many leucocytes, 66 per cent. polymorphonuclear cells. No organisms found in direct films. Culture grew pneumococei as before, but sparingly.

On the same day a fleeting erythematous rash was noticed on the face, the back, the thighs, and the buttocks; head retraction was unrelieved; the pulse-rate was slow, 60-70; the respirations were 24 ; and the temperature was $101-2^{\circ}$. On the 9th lumbar puncture was performed, when 10 c.c. of cerebro-spinal fluid were withdrawn, under greater pressure than before, and 3.5 c.c. (equivalent to 100 units) of antipneumococcal serum (Merck) were injected into the spinal canal. The injection was attended with considerable pain, especially in the legs and the back of the head; this continued for about two hours, the child tossing about and crying as though in great pain and distress. Subsequently during the evening the temperature rose to $102^{\circ}$. Two further injections of 3.5 c.c. of the serum were administered on the 10th and 11th respectively, on each occasion under an anæsthetic. On the 11 th the temperature fell to subnormal and remained so for 24 hours. On the 13th, after another rise of temperature to a maximum of $100 \cdot 4^{\circ}, 7$ c.c. (equivalent to 200 units) of the serum were injected into the spinal canal, cerebro-spinal fluid having been previously withdrawn. The painful after-effects of the injection were not diminished by the anæsthetic. The head retraction now became gradually less and the headache abated. The legs, hitherto invariably flexed, were straightened out at times. Three further injections of 7 c.c. of the serum were made on the 15th, 17th, and 19th respectively. On each occasion more cerebro-spinal fluid was withdrawn than serum injected into the spinal canal. The fluid flowed under varying pressures. The temperature still mounted on occasion to $100-101^{\circ}$, but the general curve of the chart was much flatter and the child's condition was decidedly improved, the headache and head retraction having almost disappeared. Morphinæ sulphas, gr. 1/6, was now given as soon as the child recovered from the anresthetic, and the after-effects of the injection were thereby much diminished; subsequently these were wholly prevented in the remaining serum injections by a 